\title{
Experimental discovery of Weyl semimetal TaAs
}

\author{
Xianhui Chen ${ }^{1,2}$
}

In 1929, Weyl H. proposed that the massless solution of the Dirac equation represents a pair of a new type of particles, the so-called Weyl fermions [1]. However, their existence as a fundamental particle remains evasive after more than eight decades. Neutrinos were once considered to be Weyl fermions until its mass was found. Recently, it was proposed that Weyl fermions can be hosted in a class of exotic condensed matter, so called Weyl semimetal (WSM), as quasiparticles of its low-energy excitation when two non-degenerate bands cross near Fermi level in three-dimensional momentum space [2,3]. The crossing point is called Weyl node. A Weyl node is a singularity point of Berry curvature, which can be viewed as a magnetic monopole in momentum space [2-4]. These WSMs possess pairs of Weyl nodes with opposite chirality in the bulk. One of the hallmarks in a WSM is the emergence of surface Fermi arcs, which connect the projections of Weyl nodes with opposite chiral charge on the surface $[2,3]$.

WSMs can be realized in condensed matter materials by breaking either time-reversal or inversion symmetry. Although a number of WSM candidates were theoretically proposed, the experimental realization of WSMs was impeded for many years. Until recently, a theoretical breakthrough predicted that the non-magnetic and non-centrosymmetric transition-metal monoarsenide TaAs family is WSM $[5,6]$. Compared with the previous proposals, the TaAs family realizes a WSM phase in its natural state, making experimental confirmation of WSM state easily.

In two recent online publications of Physical Review $X$ and Nature Physics, Prof. Hong Ding from the Institute of Physics, Chinese Academy of Sciences (IOP, CAS) in Beijing and his collaborators reported the experimental discovery of WSM TaAs $[7,8]$. They first measured the surface states on the (001) surface of TaAs using vacuum ultraviolet angle-resolved photoemission spectroscopy (ARPES) [7]. They developed a mathematically rigorous method to examine whether surface Fermi arcs exist. As illustrated in Fig. 1a, a closed Fermi surface (FS) can only cross an arbitrary $k$-loop in the surface Brillouin zone (BZ) an even number of times, whereas an open Fermi arc can possibly cross this loop an odd number of times. They observed that the FSs cross the closed loop $\bar{\Gamma}-\bar{X}-\bar{M}-\bar{\Gamma}$ seven times, and five times for the $\bar{\Gamma}-\bar{Y}-\bar{M}-\bar{\Gamma}$ loop (Fig. 1b), thus providing direct experimental evidence of the existence of Fermi arcs on the (001) surface of TaAs. The first-principles calculations of the surface states, which match remarkably well with the experimental results, further confirm that TaAs is a WSM. By comparing between experiment and theory, they further determined the momentum locations of Fermi arcs. Soon after, they reported the direct observation of the bulk Weyl nodes in TaAs by performing bulk-sensitive soft $\mathrm{X}$-ray ARPES measurements [8]. The projected locations at Weyl nodes onto the (001) surface BZ are connected by the Fermi arcs (Fig. 1), thus providing convincing experimental evidence that Weyl nodes in certain projected surfaces are the "source" (or "drain") for surface Fermi arcs.

Even though their experimental results have demonstrated convincingly WSM state in TaAs, much remains to be done. The TaAs family has twelve pairs of Weyl nodes near the Fermi energy $\left(E_{\mathrm{F}}\right)$, which complicates the studies of Weyl physics in them. It is appealing to search for the hydrogen atom of a WSM, a magnetic material with only two Weyl nodes at $E_{\mathrm{F}}$. Finding WSMs that emerge from time-reversal breaking would allow researchers to explore a state with properties analogous to the quantum Hall effect [4]. Moreover, additional terms can be added to Weyl Hamiltonian to obtain a new type of Weyl fermion that exhibits protected crossed FSs and has different properties to those of Weyl fermions predicted in 1929 [9]. WSMs have been expected to exhibit many anomalous transport properties, such as the negative magnetoresistance, the quantum anomalous Hall effect, the non-local quantum

\footnotetext{
${ }^{1}$ Hefei National Laboratory for Physical Sciences at Microscale and Department of Physics, University of Science and Technology of China, Hefei 230026, China

${ }^{2}$ Key Laboratory of Strongly-coupled Quantum Matter Physics of Chinese Academy of Sciences, University of Science and Technology of China, Hefei 230026, China
}

Email: chenxh@ustc.edu.cn 

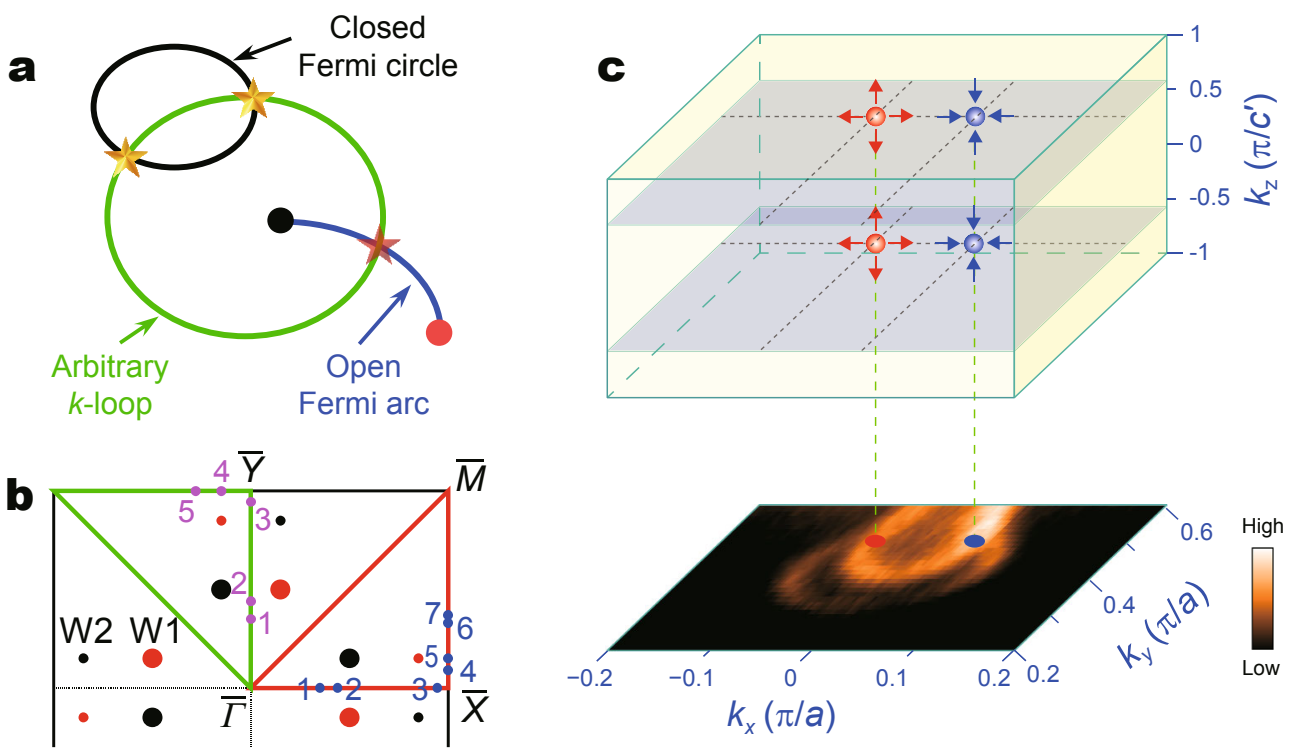

Figure 1 (a) Schematic of a closed FS pocket and an open Fermi arc crossing an arbitrary $k$-loop in the two-dimensional case. (b) Blue and magenta circles indicate the locations where the surface bands cross the enclosed $k$-loop $\bar{\Gamma}-\bar{X}-\bar{M}-\bar{\Gamma}$ and $\bar{\Gamma}-\bar{Y}-\bar{M}-\bar{\Gamma}$, respectively. (c) Locations of experimentally determined Weyl nodes in the three-dimensional BZ and their projections onto the measured (001) surface Fermi arcs. Reproduced from Ref. [7] and [8], Copyright 2015, Nature Publishing Group.

oscillation, etc. These would lead to new functionalities and applications. The experimental discovery of WSM will surely bring about immense efforts in the exciting area of physics.

Received 11 September 2015; accepted 12 September 2015; published online 18 September 2015

1 Weyl H. Electron and gravitatio.1 (in German). Z Phys, 1929, 56: 330-352

2 Wan X, Turner AM, Vishwanath A, Savrasov SY. Topological semimetal and Fermi-arc surface states in the electronic structure of pyrochlore iridates. Phys Rev B, 2011, 83: 205101

3 Xu G, Weng H, Wang Z, Dai X, Fang Z. Chern semimetal and the quantized anomalous Hall effect in $\mathrm{HgCr}_{2} \mathrm{Se}_{4}$. Phys Rev Lett, 2011,
107: 186806

4 Fang Z, Nagaosa N, Takahashi KS, et al. The anomalous Hall effect and magnetic monopoles in momentum space. Science, 2003, 302: 92-95

5 Weng H, Fang C, Fang Z, Bernevig BA, Dai X. Weyl semimetal phase in noncentrosymmetric transition-metal monophosphides. Phys Rev X, 2015, 5: 011029

6 Huang SM, Xu SY, Belopolski I, et al. A Weyl fermion semimetal with surface Fermi arcs in the transition metal monopnictide TaAs class. Nat Commun, 2015, 6: 7373

7 Lv BQ, Weng HM, Fu BB, et al. Experimental discovery of Weyl semimetal TaAs. Phys Rev X, 2015, 5: 031013

8 Lv BQ, Xu N, Weng HM, et al. Observation of Weyl nodes in TaAs. Nat Phys, 2015, 11: 724-727

9 Soluyanov AA, Gresch D, Wang Z, et al. A new type of Weyl semimetals. arXiv:1507.01603 